\title{
Plasma density inside a femtosecond laser filament in air: Strong dependence on external focusing
}

\author{
Francis Théberge, ${ }^{1}$ Weiwei Liu, ${ }^{1}$ Patrick Tr. Simard, ${ }^{1}$ Andreas Becker, ${ }^{2}$ and See Leang Chin ${ }^{1}$ \\ ${ }^{1}$ Centre d'Optique, Photonique et Laser and Département de Physique, de Génie Physique et d'Optique, Université Laval, \\ Québec, Canada, G1K $7 P 4$ \\ ${ }^{2}$ Max-Planck-Institut für Physik komplexer Systeme, Nöthnitzer Str. 38, D-01187 Dresden, Germany
}

(Received 10 March 2006; published 27 September 2006)

\begin{abstract}
Our experiment shows that external focusing strongly influences the plasma density and the diameter of femtosecond Ti-sapphire laser filaments generated in air. The control of plasma filament parameters is suitable for many applications such as remote spectroscopy, laser induced electrical discharge, and femtosecond laser material interactions. The measurements of the filament showed the plasma density increases from $10^{15} \mathrm{~cm}^{-3}$ to $2 \times 10^{18} \mathrm{~cm}^{-3}$ when the focal length decreases from $380 \mathrm{~cm}$ to $10 \mathrm{~cm}$ while the diameter of the plasma column varies from $30 \mu \mathrm{m}$ to $90 \mu \mathrm{m}$. The experimental results are in good qualitative agreement with the results of numerical simulations.
\end{abstract}

DOI: 10.1103/PhysRevE.74.036406

PACS number(s): $52.38 . \mathrm{Hb}, 52.70 . \mathrm{Kz}$

\section{INTRODUCTION}

The propagation of ultrashort and intense laser pulses in air has stimulated many research interests because of promising applications such as remote sensing of pollutants [1-7] and lightning control [8-12]. The propagation of a short intense laser pulse in a gas, such as air, is very different from that of a long pulse or continuous wave laser pulse. The mechanism for femtosecond laser pulses propagating over long distances in optical media is mainly based on the dynamic interplay between the Kerr effect due to a nonlinear intensity-dependent refractive index and defocusing from a low-density plasma that is purely induced by multiphoton/ tunnel ionization [13-16]. The plasma generation balances the self-focusing effect and leads to a limited peak intensity [17-19] along the femtosecond laser pulse propagation. This in turn creates a long (but weak) plasma column in air and results in a clean fluorescence emission (i.e., almost free from plasma continuum) of any molecule inside the filament $[1,20,21]$. The filament can persist over several tens of meters [22,23] and its formation has been observed as far as $2 \mathrm{~km}$ in the atmosphere [24]. The control of the plasma generation during the filamentation of femtosecond laser pulses will also benefit many experimental studies in spectroscopy and nonlinear optics [25-27]. There is thus a necessity to characterize the plasma channel left behind in the filaments in terms of the plasma density and the diameter. The feasibility of experiments such as the lightning discharge guidance and the remote sensing of the atmosphere critically depend on the knowledge of these plasma parameters inside the filament. However, so far, the plasma density inside the filaments in air measured by several groups $[11,28-32]$ has a wide range of values, ranging from $10^{12} \mathrm{~cm}^{-3}[11]$ to 3 $\times 10^{18} \mathrm{~cm}^{-3}$ [31] and the diameter of the plasma column ranged from $50 \mu \mathrm{m}$ [32] to $120 \mu \mathrm{m}$ [31]. These discrepancies were not the result of different measurement techniques such as the spatial interferometry or the plasma conductivity technique used by the different groups. These earlier works apparently assumed that all filaments are identical regardless of the focusing condition while unknowingly working in the multiple filamentation regime in some cases. In this work we clarify the discrepancies between the previous measurements of the plasma column and show both experimentally and numerically that the plasma density and diameter of the plasma column in the core of single filament are highly dependent on the focal length of the lens used to focus the beam but only slightly dependent on the laser power.

\section{EXPERIMENT}

Experiments were conducted using a Ti:sapphire chirped pulse amplification (CPA) laser system, which generates femtosecond laser pulses with a central wavelength at $807 \mathrm{~nm}$, a repetition rate of $10 \mathrm{~Hz}$, and a transform limited pulse duration of $45 \mathrm{fs}$ at full width at half maximum (FWHM). The radius of the beam was $4.2 \mathrm{~mm}$ at $1 / e^{2}$ of intensity. Laser pulses with energies ranging from $20 \mu \mathrm{J}$ to $4.0 \mathrm{~mJ}$ were focused into air at ambient pressure using different focal length lenses. The effective critical power for self-focusing in air $\left(P_{\mathrm{cr}}\right)$ was measured experimentally to be $10 \mathrm{GW}$ for a pulse duration of $45 \mathrm{fs}$ [33]. The focused laser pulses propagated in air and created stable filaments. All measurements were done at single filament condition; the creation of a single filament was confirmed by the observation of a single hot spot on burn papers. We characterized the plasma column of the filament using side imaging fluorescence and longitudinal diffraction techniques [34]. The setups are shown in Fig. 1(a) and the measurements and their analysis are discussed below.

\section{A. Side imaging of the plasma column}

Using an intensified charge-coupled device camera (ICCD) we imaged the fluorescence emitted from the first negative band $\left(B^{2} \Sigma_{u}{ }^{+} \rightarrow X^{2} \Sigma_{g}{ }^{+}, 391 \mathrm{~nm}\right)$ of the nitrogen ion $\left(\mathrm{N}_{2}{ }^{+}\right)$inside the plasma channel from the side $[20,21]$. The ICCD camera was installed perpendicular to the pulse propagation axis and the fluorescence signal was collected and imaged onto the ICCD detector by using a $10 \times$ microscope objective [see MO in Fig. 1(a)]. A band-pass filter together 

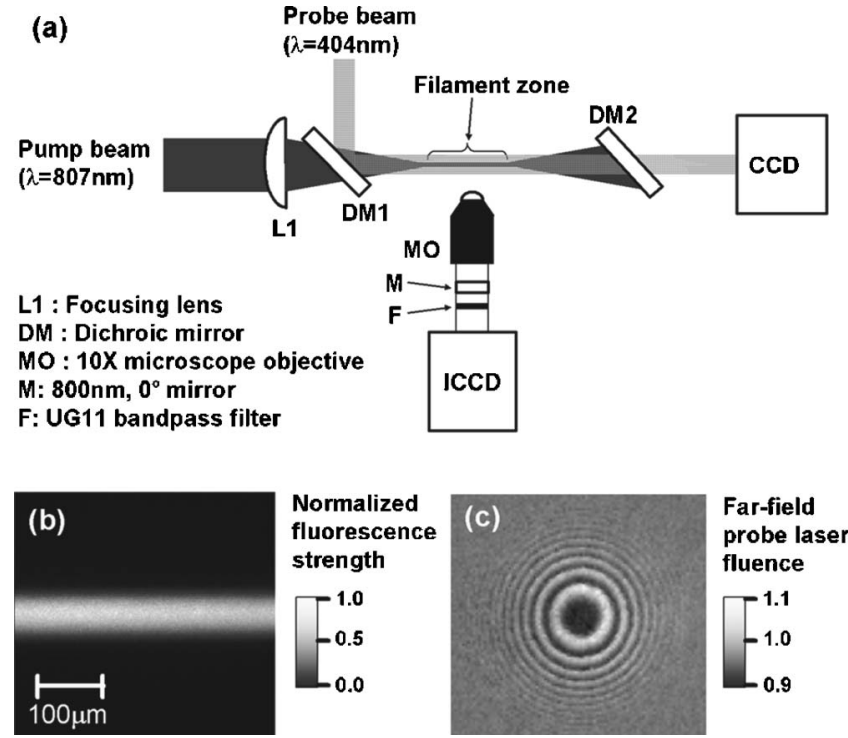

FIG. 1. (a) Experimental setup for the plasma column imaging system and the longitudinal diffraction technique. (b) Example of a plasma channel imaged from the side with the ICCD fluorescence imaging system at pump power of $1.5 P_{\mathrm{cr}}$ by using a $50 \mathrm{~cm}$ focal length lens. (c) Far-field diffraction pattern of the probe beam measured with the CCD camera for laser power of $1.5 P_{\mathrm{cr}}$ and at a delay of $330 \mathrm{fs}$ with respect to the pump pulse.

with a $0^{\circ}$ incident $800 \mathrm{~nm}$ dielectric mirror [see $\mathrm{M}$ and $\mathrm{F}$ in Fig. 1(a)] were placed in front of the camera to detect the light emission over the $\mathrm{N}_{2}{ }^{+}$band while rejecting the scattered light from the pump laser and all other emissions from nitrogen. The section of the plasma column covered by the field of view of the ICCD was $1 \mathrm{~mm}$ by $1 \mathrm{~mm}$ and the spatial resolution of $2 \mu \mathrm{m}$ was limited by the size of the ICCD's pixel. For sufficiently high pump power, the length of the plasma column in air extended over many centimeters. To characterize the plasma column over its whole length, the imaging system was scanned along the laser propagation axis and different frames were taken at each position. An example of the side imaging picture of the plasma column is shown in Fig. 1(b).

The strength of the $\mathrm{N}_{2}^{+}$fluorescence signal at $391 \mathrm{~nm}$ is directly related via the Einstein coefficient to the number of nitrogen ions in the excited $B^{2} \Sigma_{u}^{+}$state [35]. In the intensity regime of interest the number of nitrogen ions in the excited $B$ state is found to be nearly proportional to the total number of nitrogen and oxygen ions in the plasma column $[20,21,36]$. Thus, the strength of the fluorescence signal is, up to a calibration function, a measure of the plasma density. Using the side imaging technique one can therefore determine the mean and FWHM radii of the plasma column. In order to obtain the plasma density as a function of the propagation distance, the fluorescence strength was calibrated using a longitudinal diffraction technique.

\section{B. Longitudinal diffraction of a probe pulse}

The longitudinal diffraction technique was introduced in Ref. [34]. In short, it is based upon the Fraunhofer diffraction due to the phase shift $(\varphi)$ induced by the plasma column on a second harmonic probe pulse $(\lambda=404 \mathrm{~nm})$ propagating collinearly along the filament axis. The delay between the probe pulse and the pump pulse was $330 \mathrm{fs}$ in order to avoid parametric interaction with the pump pulse. An example of the far-field fluence profile of the probe pulse measured with the CCD camera is shown in Fig. 1(c).

Assuming that the probe beam has a Gaussian profile $U_{0} \propto \exp \left(-a r^{2}\right)$, the phase shift can be approximated as a cylindrical phase aperture,

$$
\varphi(r)=\frac{2 \pi}{\lambda 2 n_{c}} \int_{0}^{L} n_{e}(r, z) d z=\frac{2 \pi}{\lambda} \frac{\bar{n}_{e}(r)}{2 n_{c}} L,
$$

when $r<r_{c}$, and $\varphi=0$ when $r>r_{c}$, where $r_{c}$ is the mean radius of the plasma column, $n_{e}(z)$ is the electron density along the filament, $\bar{n}_{e}$ is the average electron density, $n_{c}$ $=\left(4 \pi^{2} c^{2} \varepsilon_{0} m_{e}\right) /\left(e^{2} \lambda^{2}\right)$ is the critical plasma density, and $L$ is the length of the plasma column [34]. Note that the length $L$ and the radius $r_{c}$ of the plasma channel are obtained using the side imaging ICCD system. The averaged plasma density $\bar{n}_{e}$ can be extracted by fitting the far-field diffraction profile $[I(r)]$ of the probe pulse after passing through the plasma channel with the results of the Fresnel diffraction integral [34],

$$
\begin{aligned}
I(r)= & -\operatorname{sgn}(K) C \pi r_{c}^{2} \frac{1}{\sqrt{a^{2}+K^{2}}} \exp \left(-b_{0} r^{2}\right) \frac{J_{1}\left(2 K r_{c} r\right)}{2 K r_{c} r} \\
& \times \cos \left(b_{1} r^{2}-\frac{\varphi(r)}{2}\right) \sin \left(\frac{\varphi(r)}{2}\right),
\end{aligned}
$$

where $d_{0}$ is the distance between the plasma filament and the CCD camera, $K=\pi / \lambda d_{0}, \quad b_{0}=K^{2} a /\left(a^{2}+K^{2}\right), \quad b_{1}=K^{3} /\left(a^{2}\right.$ $\left.+K^{2}\right), \operatorname{sgn}(K)$ is the sign of $K, J_{1}$ is the first-order Bessel function, and $C$ is a constant.

The measurements via the longitudinal diffraction technique are valid as long as the Fresnel number associated with the diameter and the length of the plasma column is large, namely $r_{c}^{2} / \lambda L>1$. The calibration was done by measurements using a $50 \mathrm{~cm}$ focal length lens and a laser pump power between $0.7 P_{\mathrm{cr}}$ and $1.5 P_{\mathrm{cr}}$ in order that the diameter and the length of the plasma column respected the condition mentioned above for the Fresnel number. These series of measurements covered a range of plasma densities between $1.7 \times 10^{15} \mathrm{~cm}^{-3}$ to $10^{16} \mathrm{~cm}^{-3}$. Using the proportionality of the $\mathrm{N}_{2}{ }^{+}$fluorescence signal to the plasma density, we compared the average fluorescence strength with the average electron density in Eq. (1) to determine the desired calibration function. As a result of the calibration, we were then able to obtain the plasma density distribution $n_{e}(z)$ directly from the $\mathrm{N}_{2}{ }^{+}$fluorescence distribution along the filament in each of the measurements. The 16 bit ICCD camera of the side imaging system gives us the possibility to measure the plasma density over more than four orders of magnitude and such a large dynamic range is not achievable with other techniques based on the spatial interferometry. The detection limit of the side imaging system was $10^{13} \mathrm{~cm}^{-3}$ and it is therefore a powerful technique to investigate in a single shot the characteristics of the self-generated plasma, namely its 
radius, length, and peak density, as well as density distribution with a spatial resolution of $2 \mu \mathrm{m}$, which was not possible with other techniques based on the spatial interferometry and the plasma conductivity.

\section{NUMERICAL SIMULATIONS}

In order to substantiate and analyze the experimental results, presented below, we have also performed numerical simulations. The filamentation in air from high-power femtosecond laser pulses involves a number of nonlinear effects such as diffraction, self-focusing, group-velocity dispersion as well as plasma generation and energy losses due to multiphoton/tunnel ionization. Our numerical model is based on the nonlinear wave equation using the slowly varying envelope approximation written in the retarded coordinate system $\tau=t-z / v_{g}(\omega)$ as $[15,37-40]$,

$$
\begin{aligned}
& 2 i k_{0} \frac{\partial A}{\partial z}+\Delta_{\perp} A-k_{2} k_{0} \frac{\partial^{2} A}{\partial \tau^{2}}+2 \frac{k_{0}^{2}}{n_{0}}\left(\Delta n_{\text {Kerr }}+\Delta n_{\text {plasma }}\right) A-i k_{0} \alpha A \\
& \quad=0 .
\end{aligned}
$$

Here, $A$ is the electric field envelope function and the nonlinear contribution to the refractive index from the neutral molecules is considered in the form $[15,37,39]$,

$$
\Delta n_{\text {Kerr }}(t)=n_{2}\left\{|A(t)|^{2}+\int_{-\infty}^{t} H\left(t-t^{\prime}\right)\left|A\left(t^{\prime}\right)\right|^{2} d t^{\prime}\right\},
$$

where $n_{2}$ is the nonlinear index of refraction. The delayed Raman response function $H(t)$ was approximated based on the damped model by the following equation:

$$
H(t)=\frac{1}{T_{k}} \theta(t) \exp \left(-t / T_{k}\right),
$$

where $\theta(t)$ is the Heaviside function and the fitting parameter $T_{k}=70 \mathrm{fs}[39,40]$. Electron generation through multiphoton/ tunnel ionization of $\mathrm{N}_{2}$ and $\mathrm{O}_{2}$ is taken into account by fitting the ionization rates into the form $\sigma I^{n}$ for the relevant intensity range, the values for $\sigma$ and $n$ are taken from Ref. [41]. Values for $v_{g}(\omega), k_{0}, k_{2}, \Delta n_{\text {plasma }}$, and $\alpha$ in air are adapted from Ref. [37]. For the numerical simulation, the self-steepening term $[38,42,43]$ and the space-time focusing [44] were not taken into account, since they are mainly responsible for the details of the spectrum and the temporal pulse shape, which are not the center of interest of this work.

We have considered the propagation of a linearly polarized, collimated Gaussian input laser pulse with a central wavelength at $\lambda_{0}=800 \mathrm{~nm}$ and a pulse duration $\tau_{F W H M}$ $=50 \mathrm{fs}$, which is focused with a lens in air. We have used laser input powers, $P_{0}$, above the critical power, $P_{\text {cr }}$ $=\lambda_{0}^{2} / 2 \pi n_{\omega} n_{2}=8 \mathrm{GW}$ needed for self-focusing in air [33] and an initial beam radius of $w_{0}=1 \mathrm{~mm}$ (at $1 / e^{2}$ of intensity).

\section{RESULTS AND DISCUSSIONS}

In Fig. 2(a) the experimentally determined effective peak plasma density is shown as a function of the laser power for four different focal length lenses and for the collimated laser
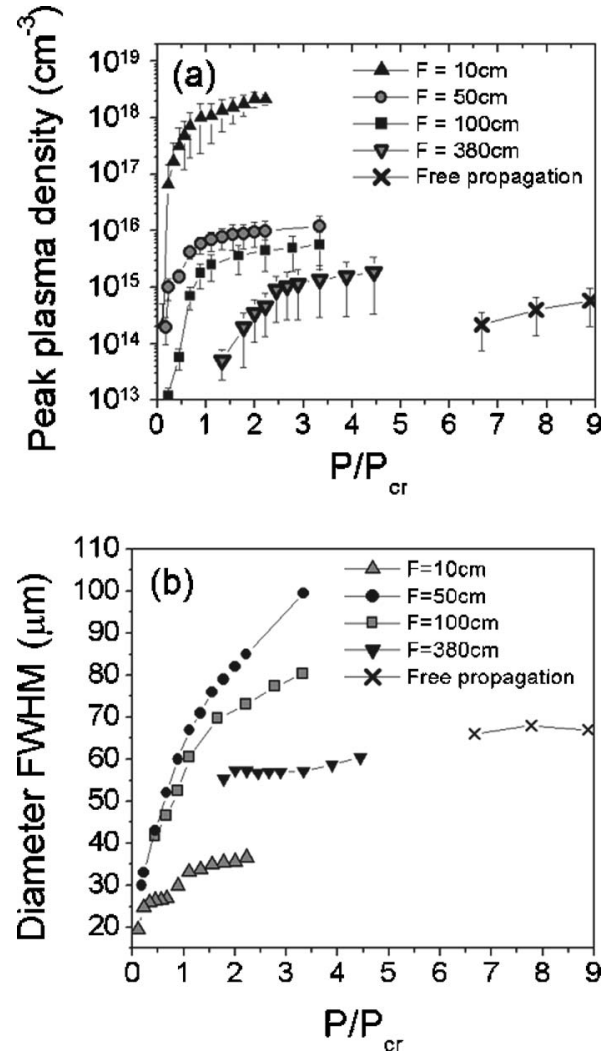

FIG. 2. (a) Effective peak plasma density and (b) FWHM plasma column diameter generated in air as a function of the initial laser power by using different focal length lenses and for the free propagating laser beam. The measurements were done with the calibrated nitrogen fluorescence imaging system.

beam. The error bars in Fig. 2(a) are due to the precision of the longitudinal diffraction technique used for the calibration of the plasma density as a function of the fluorescence from the nitrogen ions. When the lenses with focal lengths of 10 , 50 , and $100 \mathrm{~cm}$ long were used, the plasma generation occurred within the confocal region of the lenses if the pump power was lower than the critical power for self-focusing in air $\left(P_{\text {cr }}\right)$. We note that for the $380 \mathrm{~cm}$ focal length lens and a pump power below $P_{\text {cr }}$, no fluorescence could be detected by the ICCD imaging system, since the intensity at the geometrical focus was too weak and the ionization yield was too low. In case of the collimated laser beam (free propagation), the filamentation occurred far from the laser system for laser powers below $5 P_{\mathrm{cr}}$. Since our propagation experiment was limited to the scale of the lab, in this case the plasma column was characterized for pump power between $6 P_{\text {cr }}$ and $9 P_{\text {cr }}$ only.

From the results in Fig. 2(a) we observe first a steep rise of the plasma density with the increase in the input laser power before the slope changes towards a constant density at higher laser powers. A characteristic pulse peak power of about the critical power for self-focusing clearly marks the change of slope in Fig. 2(a) for the focal lengths of 10, 50, and $100 \mathrm{~cm}$. Since the plasma density depends nonlinearly on the peak intensity of the laser pulse, the steep rise at laser power below $P_{\mathrm{cr}}$ indicates a sharp increase of the laser inten- 
sity inside the confocal volume. For laser powers above $P_{\text {cr }}$ the maximum plasma density saturates for all the focal lengths as well as in the free propagation case, indicating a saturation of the peak intensity inside the filament $[17,18]$. Note also that the results clearly show that the effective peak plasma density strongly depends on the focal length. It is important to note that for similar focusing strength and laser parameters, the results shown in Fig. 2(a) are in agreement with previous measurements of the plasma filament [28-32].

Before we discuss this trend in more detail, we turn first to the experimental results for the FWHM diameter of the plasma channel at the position of the peak plasma density, presented in Fig. 2(b). We observe a strong increase of the diameter below $P_{\mathrm{cr}}$, followed by a slower increase above $P_{\mathrm{cr}}$. The increase of the plasma column diameter with the increase of the laser power was explained in Ref. [45] for a geometrical focal lens of $50 \mathrm{~cm}$. In short, the increase of the initial laser power injects more laser energy into the volume confined by the external focusing. However, the plasma generation balances the self-focusing effect and leads to a limited peak intensity $[17,18]$. Therefore, due to the counterbalance between the laser energy confined by the external focusing and the defocusing effect of the plasma, the high intensity core of the laser beam would enlarge with the increase of the initial laser power before multiple filaments form. However, as shown in Fig. 2(b), the increase of the plasma column diameter is less steep as the focal length is increased with the exception of the shortest focal length, $F$ $=10 \mathrm{~cm}$. While the diameter increases by about $50 \%$ from $60 \mu \mathrm{m}$ to $90 \mu \mathrm{m}$ between $P_{\text {cr }}$ to $3 P_{\text {cr }}$ in case of the $50 \mathrm{~cm}$ focal length lens, the increase is about $40 \%$ for the $100 \mathrm{~cm}$ focal length lens (55 $\mu \mathrm{m}$ to $77 \mu \mathrm{m})$ in the same range of initial pump power. In case of a focal length of $380 \mathrm{~cm}$ as well as for the free propagation case, we observe only a weak variation of the plasma column diameter as a function of the laser power.

In Fig. 3 we compare the experimental data [Fig. 3(a)] and the results of numerical simulations [Fig. 3(b)] for the maximum plasma density (main plot) and the FWHM diameter of the plasma column (inset) as a function of the different focal lengths. The dashed lines in the main plots and insets correspond to the value for the FWHM diameter of the plasma column and the maximum density obtained for the collimated beam at laser powers of $9 P_{\mathrm{cr}}$ (experiment) and $2.5 P_{\text {cr }}$ (simulations). As outlined above, the effective peak plasma density inside the plasma column is highly dependent on the focal length and decreases monotonically towards the limit for free propagation. We note that for a focal length of $380 \mathrm{~cm}$, the diameter and the peak density are found to be close to the corresponding values observed for the free propagating laser beam. The experimental data for the plasma density achieved with a focal length of $10 \mathrm{~cm}$ is more than four orders of magnitude larger than for the free propagation limit. The inset of Fig. 3(a) shows the experimental results for the plasma column diameter as a function of the focal length lens used. We can observe that the filament diameter was the smallest for $10 \mathrm{~cm}$ focal length lens and reached a maximum value for the $50 \mathrm{~cm}$ focal length lens while its diameter decreased to $60 \mu \mathrm{m}$ for longer focal length lenses.
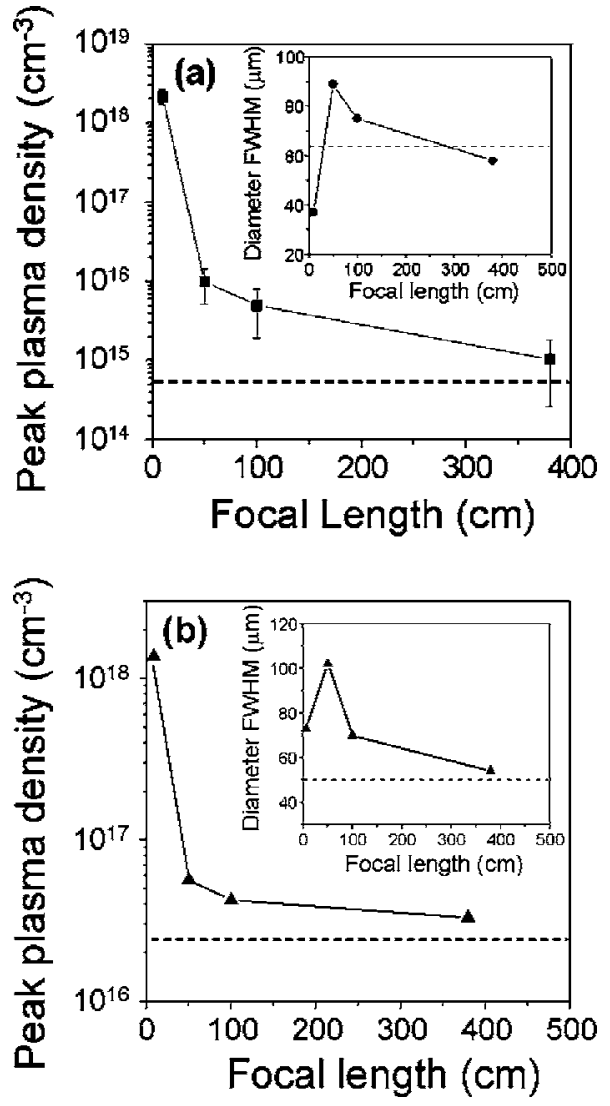

FIG. 3. (a) Experimental effective peak plasma density measured in air as a function of focal lengths of various lenses by using an initial laser power of $2.5 P_{\mathrm{cr}}$. Inset: FWHM plasma column diameter as a function of the focal lengths of the same lenses for an initial laser power of $2.5 P_{\mathrm{cr}}$. The dashed line corresponds to the plasma column parameters for the free propagating beam at laser peak power of $9 P_{\mathrm{cr}}$. (b) Numerical results of the peak plasma density in air as a function of focal lengths of various lenses by using an initial laser power of $2.5 P_{\mathrm{cr}}$. Inset: FWHM plasma column diameter as a function of the focal lengths of the same lenses for an initial laser power of $2.5 P_{\mathrm{cr}}$. The dashed line corresponds to the plasma column parameters for the free propagating beam at laser peak power of $2.5 P_{\mathrm{cr}}$.

Similarly to the experimental results, we can clearly observe in Fig. 3(b) from the results of numerical simulations that the plasma column diameter and the plasma density are dependent on the external focusing. The plasma density generated with a focal length of $6 \mathrm{~cm}$ is about two orders of magnitude larger than for the free propagating laser beam in the numerical simulations. We note also the plasma column diameter was smaller for the $6 \mathrm{~cm}$ focal length lens and reached a maximum value for an intermediate focal length of $50 \mathrm{~cm}$ while its diameter decreased again for longer focal length lenses, which is in agreement with the experimental observations. The difference between the values for the simulated and the measured characteristics of the plasma column can be explained by the smaller laser beam diameter used for the numerical simulations. Due to the large computation time needed for the numerical model with the real laser beam width $\left(w_{0}=4.2 \mathrm{~mm}\right)$, we used a smaller beam diameter for the simulation $\left(w_{0}=1 \mathrm{~mm}\right)$. Thus, as we will 


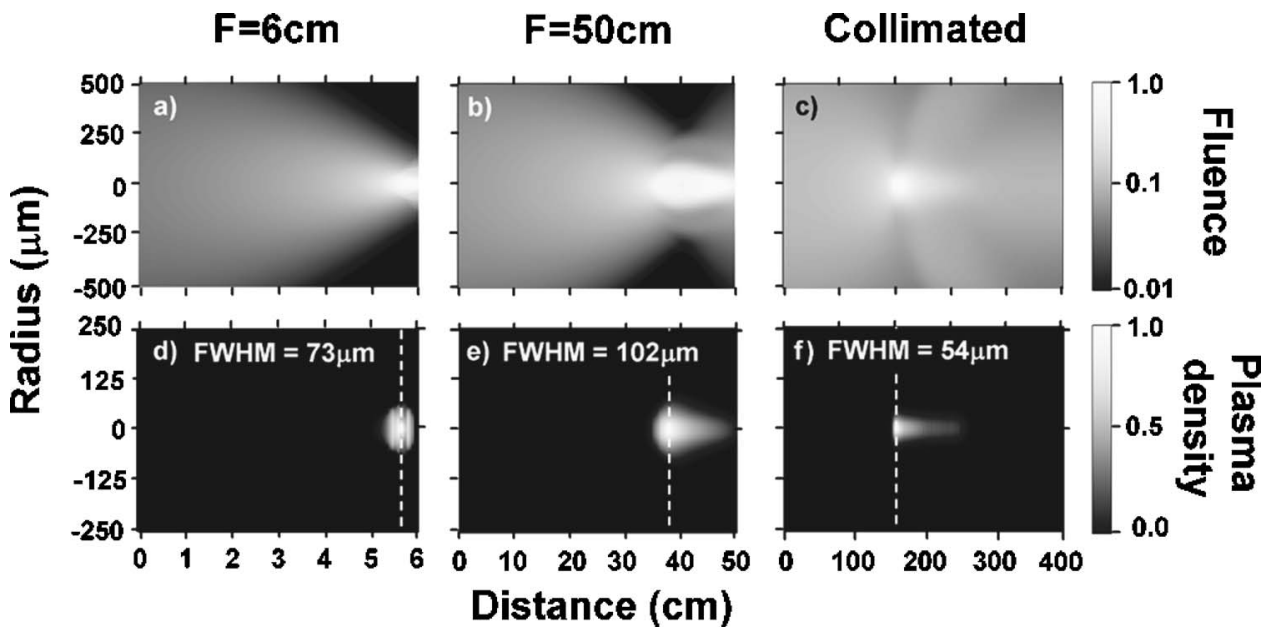

FIG. 4. Normalized laser fluence distributions (top row) and normalized electron distributions (bottom row) from the numerical simulations. (a), (d) for focal length lens of $6 \mathrm{~cm}$; (b), (e) for focal length lens of $50 \mathrm{~cm}$; (c), (f) for the collimated laser beam. The input pulse duration was $50 \mathrm{fs}$ (FWHM) and the initial laser peak power was $2.5 P_{\mathrm{cr}}$. explain below, the use of a smaller laser beam width affected the convergence and the laser intensity distribution along the propagation axis and, hence, the distribution of the selfgenerated plasma. However, as we can see in Fig. 3, the numerical simulations are in good qualitative agreement with the experimental results.

In Figs. 4(a)-4(c) we present the numerical results for the distribution of the laser fluence along the propagation axis for focal length lenses of $6 \mathrm{~cm}, 50 \mathrm{~cm}$, and for the collimated laser beam, respectively. The propagation distance is scaled from the position of the lens $(z=0)$ to the geometrical focus and over $4 \mathrm{~m}$ propagation distance for the collimated laser beam. The corresponding electron distributions are shown in Figs. 4(d)-4(f) with a normalized density scale. The dashed lines in Figs. 4(d)-4(f) indicate the position of the peak plasma density along the plasma column and the corresponding FWHM diameters are specified in the figure. Through the logarithmic scale used to represent the fluence distribution in Figs. 4(a)-4(c), we can clearly observe the laser energy confinement by the external focusing lens. For the fluence distribution of the $6 \mathrm{~cm}$ focal length lens shown in Fig. 4(a), more than $99 \%$ of the laser energy is confined within a $200 \mu \mathrm{m}$ diameter along the self-generated plasma column shown in Fig. 4(d). However, for the fluence distribution of the $50 \mathrm{~cm}$ focal length lens shown in Fig. 4(b), the laser energy was confined over a larger diameter extending up to $500 \mu \mathrm{m}$ along the self-generated plasma column; for the collimated laser beam in Fig. 4(c), the energy background extended over more than $1 \mathrm{~mm}$ diameter [46].

As shown in Figs. 4(a)-4(c), during the filamentation of an initially convergent laser beam, the laser energy is confined into a smaller volume for shorter focal length, thus increasing its intensity and, hence, the density of the selfgenerated plasma as shown in Fig. 3. By using the semiempirical model for the rate of tunnel ionization of $\mathrm{N}_{2}$ and $\mathrm{O}_{2}$ molecules [41], we estimated from the measured plasma density in Fig. 3(a) that the laser intensity in the core of the filament for the $10 \mathrm{~cm}$ focal length lens was 1.6 $\times 10^{14} \mathrm{~W} / \mathrm{cm}^{2}$ for initial laser peak power of $2.5 P_{\mathrm{cr}}$. For the $380 \mathrm{~cm}$ focal length lens, the estimated laser intensity in the core of the filament was $5.3 \times 10^{13} \mathrm{~W} / \mathrm{cm}^{2}$ for the same initial peak power of $2.5 P_{\mathrm{cr}}$. This clearly shows that the peak laser intensity increases with stronger external focusing.
However, the laser intensity changes by a factor of 3 only, but due to the highly nonlinear intensity dependence of the plasma generation, the density of the self-generated plasma changes by more than four orders of magnitude [see Figs. 2(a) and 3(a)]. The dependence of the laser intensity reached at the core of the filament with the external focusing can be summarized as follows: (1) By using a shorter focal length lens, the laser energy is confined into a smaller volume, as shown in Figs. 4(a)-4(c) resulting in a higher intensity at the self-focus when the laser pulse self-focuses. (2) For the same laser peak power and by using a shorter focal length lens, the convergence of the laser pulse increases due to the stronger external focusing. Thus, a higher plasma density is needed to counterbalance the stronger convergence of the pulse.

The dependence of the plasma column diameter on the external focal length [see Fig. 2(b) and insets in Fig. 3] can be explained as a balance between the laser energy confinement by the external focusing, the self-focusing, and the defocusing effect of the self-generated plasma. For a long focal length lens or for the initially collimated laser beam, the diameter of the plasma column is mainly governed by the dynamic equilibrium between the self-focusing in air and defocusing by the self-generated plasma. In this case, as we can observe in Figs. 4(c) and 4(f), the plasma generation occurs essentially in the high intensity core of the laser beam cross section and the observed diameter of the plasma column is $54 \mu \mathrm{m}$ at FWHM. For intermediate focal length lenses (between $50 \mathrm{~cm}$ to $380 \mathrm{~cm}$ ), external focusing concentrates more laser energy on the propagation axis and the high intensity core at the self-focus is enlarged. In this case, the diameter of the self-generated plasma column increases with the decrease of the focal length and reaches a maximum diameter for the $50 \mathrm{~cm}$ focal length lens. Beyond this point, with shorter focal length lenses, the diameter of the self-focal zone can no longer increase because the geometrical focal diameter becomes smaller. That is to say, external focusing becomes dominant and there is practically no distinction between geometrical focusing and self-focusing. Therefore, the diameter of the laser beam is limited by the strong external focusing of the lens, as observed in insets of Fig. 3 where the plasma column diameter decreases for shorter focal length lens. 


\section{CONCLUSIONS}

Our experimental observations and numerical simulations clarify the discrepancies between the previous measurement of the plasma density and the plasma column diameter $[11,28-32]$ by using different focal length lenses and laser power. With the use of the calibrated side imaging technique, we observed that the plasma column parameters and the laser intensity in the filament are strongly dependent on the external focusing and slightly dependent on the initial laser power. For the free propagating laser beam, we measured experimentally an initial self-generated plasma density of $4.5 \times 10^{14} \mathrm{~cm}^{-3}$ and the laser intensity inside the filament was estimated to be $5 \times 10^{13} \mathrm{~W} / \mathrm{cm}^{2}$. Finally looking ahead, the use of the optimum focal length lens and laser beam diameter should improve the efficiency of many nonlinear intensity dependent processes based upon the propagation of ultrashort and intense laser pulses. For example, the conversion efficiency of high-harmonics generation in a gas cell or the characteristic fluorescence strength from the ionization of pollutants using remote sensing technique could be increased by optimizing the volume and the laser intensity of the generated filament.

\section{ACKNOWLEDGMENTS}

We acknowledge the support in part by Natural Sciences and Engineering Research Council of Canada (NSERC), Defence R\&D Canada-Valcartier (DRDC-Valcartier), Le Fonds Québécois de la Recherche sur la Nature et les Technologies (FQRNT), Canada Research Chairs (CRC), Canada Foundation for Innovation (CFI), and Canadian Institute for Photonic Innovations (CIPI). We appreciate the technical support of M. Mario Martin.
[1] J.-F. Gravel, Q. Luo, D. Boudreau, X. P. Tang, and S. L. Chin, J. Anal. Chem. USSR 76, 4799 (2004).

[2] J. Kasparian, M. Rodriguez, G. Méjean, J. Yu, E. Salmon, H. Wille, R. Bourayou, S. Frey, Y.-B. André, A. Mysyrowicz, R. Sauerbrey, J.-P. Wolf, and L. Wöste, Science 301, 61 (2003).

[3] P. Rairoux, H. Schillinger, S. Niedermeier, M. Rodriguez, F. Ronneberger, R. Sauerbrey, B. Stein, D. Waite, C. Wedekind, H. Wille, L. Wöste, and C. Ziener, Appl. Phys. B: Lasers Opt. 71, 573 (2000).

[4] L. Wöste, C. Wedekind, H. Wille, P. Rairoux, B. Stein, S. Nikolov, C. Werner, S. Niedermeier, F. Ronneberger, H. Schillinger, and R. Sauerbrey, Laser Optoelektron. 29, 51 (1997).

[5] H. L. Xu, J. F. Daigle, Q. Luo, and S. L. Chin, Appl. Phys. B: Lasers Opt. 82, 655 (2006).

[6] Q. Luo, H. L. Xu, S. A. Hosseini, J.-F. Daigle, F. Théberge, M. Sharifi, and S. L. Chin, Appl. Phys. B: Lasers Opt. 82, 105 (2006).

[7] Q. Luo, S. A. Hosseini, B. Ferland, and S. L. Chin, Opt. Commun. 233, 411 (2004).

[8] H. Pépin, D. Comptois, F. Vidal, C. Y. Chien, A. Desparois, T. W. Johnston, J. C. Kieffer, B. L. Fontaine, F. Martin, F. A. M. Rizk, C. Potvin, P. Couture, H. P. Mercure, A. BondiouClergerie, P. Lalande, and I. Gallimberti, Phys. Plasmas 8, 2532 (2001).

[9] M. Rodriguez, R. Sauerbrey, H. Wille, L. Wöste, T. Fujii, Y.-B. André, A. Mysyrowicz, L. Klingbeil, K. Rethmeier, W. Kalkner, J. Kasparian, E. Salmon, J. Yu, and J.-P. Wolf, Opt. Lett. 27, 772 (2002).

[10] X. M. Zhao, J.-C. Diels, C. Y. Wang, and J. M. Elizondo, IEEE J. Quantum Electron. 31, 599 (1995).

[11] H. Schillinger and R. Sauerbrey, Appl. Phys. B: Lasers Opt. 68, 753 (1999).

[12] S. L. Chin and K. Miyazaki, Jpn. J. Appl. Phys., Part 1 38, 2011 (1999).

[13] N. Aközbek, C. M. Bowden, and S. L. Chin, J. Mod. Opt. 49, 475 (2002).

[14] A. Brodeur, C. Y. Chien, F. A. Ilkov, S. L. Chin, O. G. Kosa- reva, and V. P. Kandidov, Opt. Lett. 22, 304 (1997).

[15] M. Mlejnek, E. M. Wright, and J. V. Moloney, Opt. Lett. 23, 382 (1998).

[16] S. L. Chin, S. A. Hosseini, W. Liu, Q. Luo, F. Théberge, N. Aközbek, A. Becker, V. P. Kandidov, O. G. Kosareva, and H. Schroeder, Can. J. Phys. 83, 863 (2005).

[17] A. Becker, N. Aközbek, K. Vijayalakshmi, E. Oral, C. M. Bowden, and S. L. Chin, Appl. Phys. B: Lasers Opt. 73, 287 (2001).

[18] J. Kasparian, R. Sauerbrey, and S. L. Chin, Appl. Phys. B: Lasers Opt. 71, 877 (2000).

[19] W. Liu, S. Petit, A. Becker, N. Akozbek, C. M. Bowden, and S. L. Chin, Opt. Commun. 202, 189 (2002).

[20] A. Talebpour, S. Petit, and S. L. Chin, Opt. Commun. 171, 285 (1999).

[21] A. Talebpour, M. Abdel-Fattah, A. D. Bandrauk, and S. L. Chin, Laser Phys. 11, 68 (2001).

[22] G. Méchain, C. D’Amico, Y.-B. André, S. Tzortzakis, M. Franco, B. Prade, A. Mysyrowicz, A. Couairon, E. Salmon, and R. Sauerbrey, Opt. Commun. 247, 171 (2005).

[23] A. Brodeur, C. Y. Chien, F. A. Ilkov, S. L. Chin, O. G. Kosareva, and V. P. Kandidov, Opt. Lett. 22, 304 (1997).

[24] M. Rodriguez, R. Bourayou, G. Méjean, J. Kasparian, J. Yu, E. Salmon, A. Scholz, B. Stecklum, J. Eislöffel, U. Laux, A. P. Hatzes, R. Sauerbrey, L. Wöste, and J.-P. Wolf, Phys. Rev. E 69, 036607 (2004).

[25] H. L. Xu, W. Liu, and S. L. Chin, Opt. Lett. 31, 1540 (2006).

[26] A. Couairon, M. Franco, A. Mysyrowicz, J. Biegert, and U. Keller, Opt. Lett. 30, 2657 (2005).

[27] J. R. Sutherland, E. L. Christensen, N. D. Powers, S. E. Rhynard, J. C. Painter, and J. Peatross, Opt. Express 12, 4430 (2004).

[28] S. Tzortzakis, B. Prade, M. Franco, and A. Mysyrowicz, Opt. Commun. 181, 123 (2000).

[29] B. La Fontaine, F. Vidal, Z. Jiang, C. Y. Chien, D. Comtois, A. Desparois, T. W. Johnston, J.-C. Kieffer, H. Pépin, and H. P. Mercure, Phys. Plasmas 6, 1615 (1999).

[30] C. Y. Chien, B. La Fontaine, A. Desparois, Z. Jiang, T. W. 
Johnston, J. C. Kieffer, H. Pépin, F. Vidal, and H. P. Mercure, Opt. Lett. 25, 578 (2000).

[31] H. Yang, J. Zhang, Y. Li, J. Zhang, Y. Li, Z. Chen, H. Teng, Z. Wei, and Z. Sheng, Phys. Rev. E 66, 016406 (2002).

[32] A. Ting, I. Alexeev, D. Gordon, R. Fischer, D. Kaganovich, T. Jones, E. Briscoe, J. Peñano, R. Hubbard, and P. Sprangle, Phys. Plasmas 12, 056705 (2005).

[33] W. Liu and S. L. Chin, Opt. Express 13, 5750 (2005).

[34] J. S. Liu, Z. L. Duan, Z. N. Zeng, X. H. Xie, R. X. Li, S. L. Chin, and Z. Z. Xu, Phys. Rev. E 72, 026412 (2005).

[35] E. Arévalo and A. Becker, Phys. Rev. A 72, 043807 (2005).

[36] A. Becker, A. D. Bandrauk, and S. L. Chin, Chem. Phys. Lett. 343, 345 (2001).

[37] V. P. Kandidov, O. G. Kosareva, I. S. Golubtsov, W. Liu, A. Becker, N. Akozbek, C. M. Bowden, and S. L. Chin, Appl. Phys. B: Lasers Opt. 77, 149 (2003).

[38] N. Aközbek, M. Scalora, C. M. Bowden, and S. L. Chin, Opt.
Commun. 191, 353 (2001).

[39] A. Chiron, B. Lamouroux, R. Lange, J.-F. Ripoche, M. Franco, B. Prade, G. Bonnaud, G. Riazuelo, and A. Mysyrowicz, Eur. Phys. J. D 6, 383 (1999).

[40] J.-F. Ripoche, G. Grillon, B. Prade, M. Franco, E. Nibbering, R. Lange, and A. Mysyrowicz, Opt. Commun. 135, 310 (1997).

[41] A. Talebpour, J. Yang, and S. L. Chin, Opt. Commun. 163, 29 (1999).

[42] F. DeMartini, C. H. Townes, T. K. Gustafson, and P. L. Kelley, Phys. Rev. 164, 312 (1967).

[43] A. L. Gaeta, Phys. Rev. Lett. 84, 3582 (2000).

[44] J. E. Rothenberg, Opt. Lett. 17, 1340 (1992).

[45] Y. P. Deng, J. B. Zhu, Z. G. Ji, J. S. Liu, B. Shuai, R. X. Li, Z. Z. Xu, F. Théberge, and S. L. Chin, Opt. Lett. 31, 546 (2006).

[46] W. Liu, F. Théberge, E. Arévalo, J.-F. Gravel, A. Becker, and S. L. Chin, Opt. Lett. 30, 2602 (2005). 